\title{
Effects of nuclear factor- $\kappa B$ and ERK signaling transduction pathway inhibitors on human melanoma cell proliferation in vitro
}

\author{
YI-CHUAN HUANG ${ }^{1 *}$, MIN PAN $^{2}$, NING LIU $^{3}$, JUN-GANG XIAO $^{2}$ and HONG-QUAN CHEN ${ }^{2 *}$ \\ Departments of ${ }^{1}$ Otorhinolaryngology and ${ }^{2}$ Dermatology, Affiliated Hospital of Qingdao University, Qingdao, \\ Shandong 266003; ${ }^{3}$ Department of Dermatology, Qingdao Municipal Hospital, Qingdao, Shandong 266000, P.R. China
}

Received September 19, 2014; Accepted July 21, 2015

DOI: 10.3892/ol.2015.3672

\begin{abstract}
The present study aimed to investigate the effects of blocking nuclear factor (NF)- $\kappa \mathrm{B}$ and/or extracellular signal-regulated kinase (ERK) signaling pathways on proliferation and apoptosis of melanoma cells in vitro. A375 Human melanoma cells were treated with U0126 (ERK signaling pathway inhibitor) and BMS-345541 (NF- $\mathrm{BB}$ inhibitor), alone or in combination. At 12, 24 and $48 \mathrm{~h}$ after treatment, cell viability was assessed using the 3-(4,5-dimethylthiazol-2-yl)-2,5-diphenyltetrazolium bromide assay, cell cycle progression and apoptosis were evaluated by flow cytometry, and Bcl-2 protein content was determined by western blot analysis. BMS-345541 and U0126 significantly inhibited A375 cell proliferation in a dose- and time-dependent manner $(\mathrm{P}<0.01)$. The rate of proliferation inhibition at $24 \mathrm{~h}$ was $35.41 \pm 1.38 \%$ for BMS-345541 alone, $30.64 \pm 2.86 \%$ for U0126 alone, and $77.27 \pm 2.70 \%$ for BMS-345541 and U0126 in combination. The difference between combination and single treatment was significantly different $(\mathrm{P}<0.01)$. The proportion of cells in $\mathrm{S}$ phase was $14.20,18.40$ and $22.64 \%$ following treatment with BMS-345541, U0126, and BMS-345541 and U0126 in combination, respectively; these values were all significantly reduced compared with the untreated control group $(\mathrm{P}<0.01)$. The apoptosis rate was $24.98 \pm 1.03 \%$ in the BMS-345541 group, $13.96 \pm 0.96 \%$ in the U0126 group and $38.91 \pm 1.46 \%$ in the combination group; all significantly increased compared with the control group $(\mathrm{P}<0.01)$. Bcl-2 protein content in A375 cells was significantly increased following treatment with BMS-345541 and U0126, alone or in combination, when compared with the untreated
\end{abstract}

Correspondence to: Dr Hong-Quan Chen, Department of Dermatology, Affiliated Hospital of Qingdao University, 16 Jiangsu Road, Qingdao, Shandong 266003, P.R. China

E-mail: chhq6198@163.com

*Contributed equally

Key words: nuclear factor- $\kappa \mathrm{B}$, extracellular signal-regulated kinase, melanoma control group $(\mathrm{P}<0.01)$. Therefore, $\mathrm{NF}-\kappa \mathrm{B}$ and ERK signaling pathway inhibitors may serve as potential therapeutic targets for melanoma.

\section{Introduction}

Malignant melanoma is the most aggressive type of skin cancer, and its incidence and mortality have increased steadily over the last 50 years to $3 \%$ of all tumors (1). Prognosis is poor once melanoma has metastasized, with a median survival time of $4-6$ months $(1,2)$. The B-Raf inhibitor dabrafenib, in combination with MEK inhibitor trametinib were approved in 2013 by the Food and Drug Administration as a treatment strategy for unresectable or metastatic BRAF-mutated melanoma. Compared with BRAF-inhibitor monotherapy, combined therapy offers an improved response rate for the treatment of advanced melanoma. However, similar to monotherapy, associated toxicity and tumor resistance and progression are still observed in the majority of patients (3).

Previous studies have revealed that activating BRAF kinase mutations drive oncogenesis in a wide variety of malignancies, most notably so in melanoma ( $70 \%$ of cases). These mutations reduce the activation state of the Raf-MEK-ERK mitogen-activated protein kinase signalling pathway, which is involved in the growth of Raf-mutated melanoma (4), and B-Raf has been exploited as a novel therapeutic target for melanoma in recent years. The present authors have previously demonstrated that the MEK/ERK inhibitor U0126 is capable of inhibiting the proliferation of the A375 human malignant melanoma cell line in vitro. However, the cells develop resistance to U0126, preventing a durable response (5), a phenomenon shared with other MEK/ERK inhibitors (6). Various studies have demonstrated that inhibiting the nuclear factor (NF)- $\kappa \mathrm{B}$ signaling pathway increases the cytotoxicity of anticancer agents, thus, reducing multiple drug resistance in the tumor $(7,8)$. However, there are no reports on the synergistic effect of MEK/ERK and $N F-\kappa B$ inhibitors on melanoma cell proliferation in the current literature.

The aim of the current study is to evaluate the effects of the NF- $\kappa \mathrm{B}$ inhibitor BMS-35541 and the MEK/ERK inhibitor $\mathrm{U} 0126$, alone or in combination, on the proliferation and apoptosis of human melanoma cells in vitro. This may increase the field of applications for MEK-targeted therapy. 


\section{Materials and methods}

Cell culture. The A375 human malignant melanoma cell line was purchased from the Cell Bank of the Chinese Academy of Sciences (Shanghai, China). The cells were cultured in Dulbecco's modified Eagle's medium (GE Healthcare Life Sciences, Logan, UT, USA) and supplemented with $10 \%$ fetal bovine serum (FBS; GE Healthcare Life Sciences, Little Chalfont, UK), $100 \mathrm{U}$ penicillin and $100 \mu \mathrm{g}$ streptomycin at $37^{\circ} \mathrm{C}$ in a humid atmosphere of $5 \% \mathrm{CO}_{2}$. The study protocol was approved by Ethics Committee of the Affiliated Hospital of Qingdao University (Qingdao, China).

Proliferation inhibition assay. A375 cells were seeded into 96-well plates at a density of 1,000 cells/well. Following incubation in 10\% FBS medium for $24 \mathrm{~h}$, the cells were then incubated with either $150 \mu \mathrm{l} /$ well dimethyl sulphoxide (DMSO; vehicle control), the ERK inhibitor U0126 (1,5 or $10 \mu \mathrm{mol} / \mathrm{l})$, the NF- $\kappa$ B inhibitor BMS-345541 (1,5 or $10 \mu \mathrm{mol} / \mathrm{l})$, or U0126 and BMS-345541 in combination ( $5 \mu \mathrm{mol} / \mathrm{l})$. At timepoints of 12,24 , 48 and $72 \mathrm{~h}$ following treatment, cell viability was assessed using the 3-(4,5-dimethylthiazol-2-yl)-2,5-diphenyl-tetrazolium bromide (MTT) assay (9). Optical density values were determined and the rate of inhibition of cellular proliferation was calculated using the following equation: $\left(1\right.$ - mean $\left.\mathrm{OD}_{\text {experimental group }}\right) /\left(\right.$ mean $\left.\mathrm{OD}_{\text {control group }}\right) \times 100 \%$ (9). The combined effect of U0126 and BMS-345541 was determined as previously described (10). DMSO, U0126, BMS-345541 and MTT were all purchased from Sigma-Aldrich (St. Louis, MO, USA). The proliferation inhibition assay was performed three times.

Flow cytometry. A 375 cells were seeded into six-well plates and treated with inhibitors, as described in the proliferation assay. At 12, 24, 48 and $72 \mathrm{~h}$ after treatment, cells were harvested and stained with fluorescein isothiocyanate-labeled annexin V and propidium iodide (PI; Sigma-Aldrich). Cell cycle progression and apoptosis was analyzed using a FACSCalibur ${ }^{\mathrm{TM}}$ flow cytometer and CellQuest ${ }^{\mathrm{TM}}$ software (BD Biosciences, San Jose, CA, USA).

Western blotting. A375 cells were plated onto dishes and treated with various inhibitors, as described in the proliferation assay. At the end of the designated culture period, cells were washed twice in ice-cold phosphate-buffered saline (PBS) and then lyzed in ice-cold radioimmunoprecipitation assay lysis buffer. Cell homogenates were obtained following the removal of non-soluble debris by centrifugation at a high speed for $20 \mathrm{~min}$ at $4^{\circ} \mathrm{C}$. Homogenized proteins $(20 \mu \mathrm{g})$ were loaded into a $12 \%$ polyacrylamide gel, separated by SDS-PAGE and transferred onto a nitrocellulose membrane using a Transblot ${ }^{\circledR}$ apparatus at $100 \mathrm{~V}$ for $90 \mathrm{~min}$. The membrane was sequentially incubated with polyclonal mouse anti-human Beclin-2 (cat no. ZM-0010; 1:400 dilution) and monoclonal mouse anti-human GAPDH (cat no. ab8245; 1:1,000 dilution) primary antibodies, followed by horseradish peroxidase-conjugated goat anti-mouse IgG (cat no. ZDR-5307; 1:10,000 dilution) secondary antibody. All antibodies used were diluted in Tris-buffered saline with Tween 20 and purchased from OriGene Technologies, Inc.
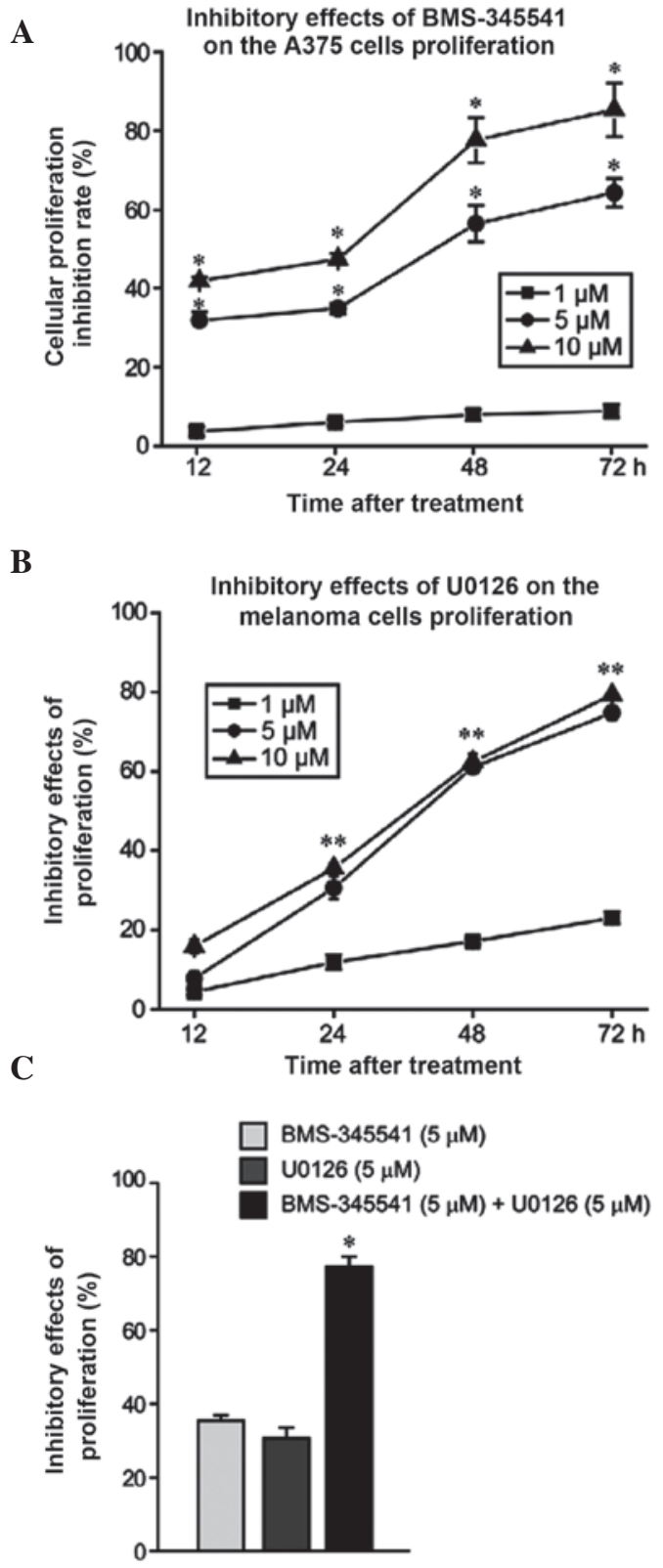

Figure 1. MTT assay demonstrating the ability of (A) BMS-345541 and (B) U0126, alone or (C) in combination to inhibit A375 cell proliferation at indicated doses and time points. Error bars indicate mean \pm standard deviation. ${ }^{*} \mathrm{P}<0.05$

(Beijing, China). The membranes were washed with PBS three times for 10 min during and after immunolabelling. Protein expression was quantified using Image J software (National Institutes of Health, Bethesda, MD, USA).

Statistical analysis. Data were analyzed by one-way analysis of variance and Student's t-test, using SPSS version 10.0 statistical software (SPSS Inc., Chicago, IL, USA). P $<0.05$ was considered to represent a statistically significant difference.

\section{Results}

Cell viability. At all timepoints, BMS-345541 demonstrated a significantly higher ability to suppress A375 cell proliferation when used at 5 and $10 \mu \mathrm{mol} / 1$ compared with $1 \mu \mathrm{mol} / 1(\mathrm{P}<0.05$, 

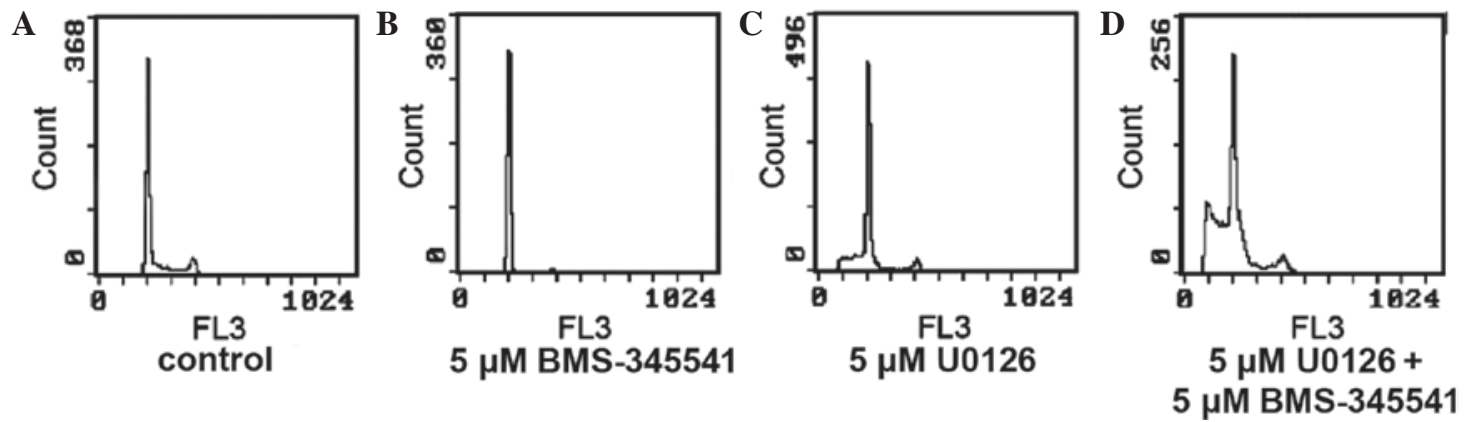

Figure 2. Representative flow cytometry histograms of cell cycle progression in (A) untreated (control) A375 cells and (B) cells treated with $5 \mu$ mol/1 BMS-345541, and (C) $5 \mu \mathrm{mol} / 1 \mathrm{U} 0126$ and (D) $5 \mu \mathrm{mol} / 1 \mathrm{BMS}-345541$ combined with $5 \mu \mathrm{mol} / 1 \mathrm{U} 0126$.
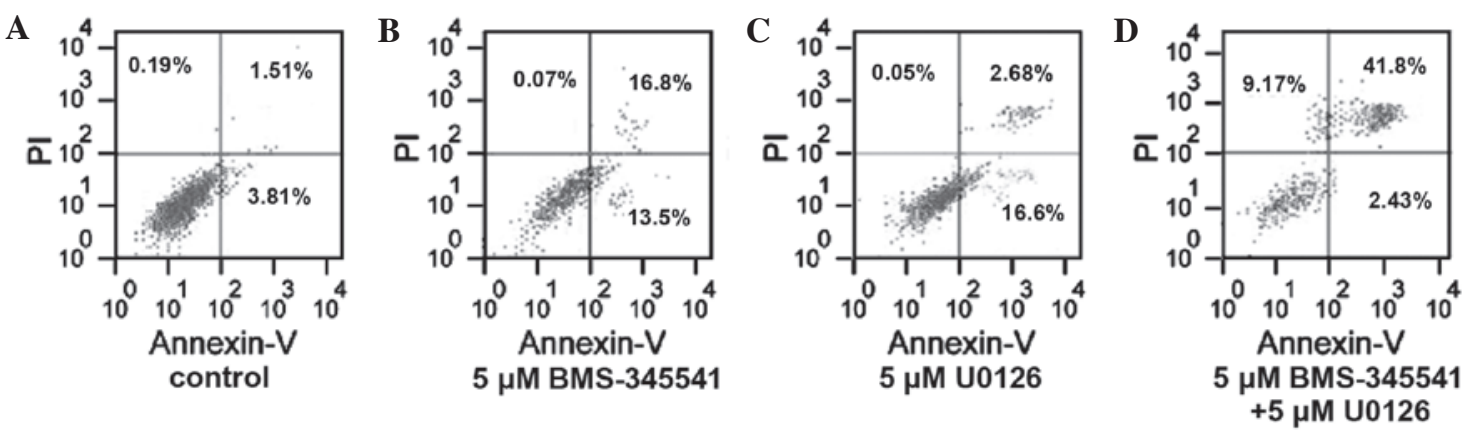

Figure 3. Apoptosis of A375 cells in each group treated for 24 h. (A) Control group; (B) BMS-345541 (5 $\mu$ mol/l); (C) U0126 (5 $\mu$ mol/l) and (D) combination $(5 \mu \mathrm{mol} / 1 \mathrm{BMS}-345541+5 \mu \mathrm{mol} / 1 \mathrm{U} 0126)$. The apoptosis rate of the combination group was significantly higher than that in the BMS-345541 and U0126 groups $(\mathrm{P}<0.01)$.

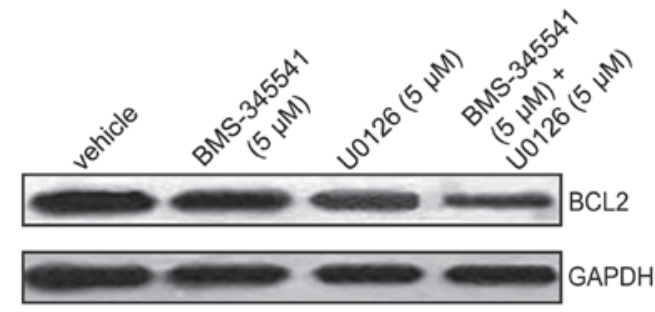

Figure 4. Effect of two drugs on Bcl-2 expression in A375 cells: Control untreated group; BMS-345541 (5 $\mu \mathrm{mol} / \mathrm{l})$; U0126 (5 $\mu \mathrm{mol} / 1)$ and combination (5 $\mu \mathrm{mol} / 1 \mathrm{BMS}-345541+5 \mu \mathrm{mol} / 1 \mathrm{U} 0126)$ group.

Fig. 1A). By contrast, the inhibitory effect of U0126 was not significantly different between 5 and $10 \mu \mathrm{mol} / 1$ and $1 \mu \mathrm{mol} / 1$ at $12 \mathrm{~h}(\mathrm{P}>0.05)$, but became significantly different at 24 , 48 and $72 \mathrm{~h}(\mathrm{P}<0.05$; Fig. 1B). The half maximal inhibitory concentration at $24 \mathrm{~h}$ was $\sim 5 \mu \mathrm{mol} / 1$ for both drugs. When BMS-345541 and U0126 were used in combination, the inhibition rate of cell proliferation was significantly higher than when they were used alone $(\mathrm{P}<0.05$; Fig. 1C).

Cell cycle progression. Compared with the untreated controls (Fig. 2A), treatment with BMS-345541 for $24 \mathrm{~h}$ (Fig. 2B) significantly increased the proportion of A375 cells in G1 $(62.97 \%$ vs. $69.13 ; \mathrm{P}<0.01)$ and $\mathrm{G} 2(15.6$ vs. $7.57 \%$; $\mathrm{P}<0.01)$, but there was a significant reduction in the proportion of cells in $\mathrm{S}$ phase $(29.47 \%$ vs. 15.27 ; $\mathrm{P}<0.01)$. U0126 significantly increased the percentage of cells in G1 phase $(84.80$ vs. $62.97 \% ; \mathrm{P}<0.01)$ and decreased the

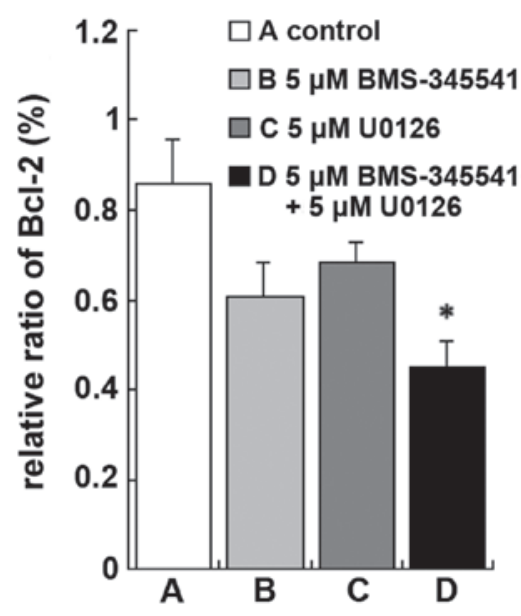

Figure 5. Quantification of Bcl-2 protein expression levels in each group. ${ }^{*} \mathrm{P}<0.01$ vs. group A (control).

percentage of cells in $\mathrm{S}$ phase $(11.07$ vs. $29.47 \%$; $\mathrm{P}<0.01)$ and $\mathrm{G} 2$ phase (4.13 vs. $7.57 \%$; $\mathrm{P}>0.01$ ). In the combination group, the proportion of cells in G1 phase fell between those of the BMS-345541 group and the U0126 group $(73.43 \%$; $\mathrm{P}<0.01)$; the percentage of G2 phase cells was marginally higher than in the BMS-345541 group (16.3 vs. $15.6 \%)$; and the percentage of $\mathrm{S}$ phase cells reduced to $6.83 \%(\mathrm{P}<0.01)$.

Cell apoptosis. Apoptosis was induced more frequently in the A375 cells treated by BMS-345541 in combination with U0126 than when used with either inhibitor alone. 
The apoptosis rate in the BMS-345541 (5 $\mu \mathrm{mol} / \mathrm{l})$ and U0126 (5 $\mu \mathrm{mol} / \mathrm{l})$ increased by $24.98 \%(\mathrm{t}=9.74, \mathrm{P}<0.01)$ and $13.96 \%(\mathrm{t}=15.82, \mathrm{P}<0.01)$, respectively, compared with the control group. Furthermore, the apoptosis rate of the combination group ( $5 \mu \mathrm{mol} / 1 \mathrm{BMS}-345541$ plus $5 \mu \mathrm{mol} / 1 \mathrm{U} 0126)$ increased by $38.91 \%$, which was significantly more than that of the control group $(\mathrm{t}=8.15, \mathrm{P}<0.01$; Fig. 3$)$.

Changes in Bcl-2 protein expression levels. To further explore the mechanism by which BMS-345541 and U0126 induce A375 cell apoptosis, western blotting was used to measure the protein expression levels of Bcl-2, a known regulator of apoptosis. Treatment with BMS-345541, in combination with U0126 resulted in a significant reduction in $\mathrm{Bcl}-2$ protein expression compared with the control, BMS-345541 (5 $\mu \mathrm{mol} / \mathrm{l})$ and U0126 (5 $\mu \mathrm{mol} / \mathrm{l})$ (Figs. 4 and 5 ; $\mathrm{P}<0.01)$. These results indicate that BMS-345541 in combination with U0126 strongly inhibited proliferation of A 375 cells by activating the intrinsic apoptosis signaling pathway.

\section{Discussion}

The Raf/MEK/ERK protein kinase cascade is an important intracellular signaling pathway that influences a number of fundamental cellular processes. Aberrant activation of the pathway is a major cause of cancer cell growth (11). Ras, a member of this protein network is mutated and active in $~ 30 \%$ of all cases of cancer and B-Raf is the most commonly mutated kinase in human cancer $(70 \%$ of melanomas) $(11,12)$. MEK inhibition is consequently an important and logical target, however, proof of concept has yet to be identified in clinical trials. In accordance with previous studies, the results of the current study demonstrated that treatment with the MEK inhibitor U0126 resulted in greater induction of A375 melanoma cell apoptosis $(13,14)$. However, the invariable development of resistance to these agents (including U0126 and other MEK inhibitors) represents a significant clinical obstacle to their long-term efficacy $(6,13,14)$.

Activation of $\mathrm{NF}-\kappa \mathrm{B}$ is considered to confer resistance to cytotoxic therapies and allow an escape from apoptosis. The inhibitor of $\kappa \mathrm{B}$ kinase complex (IKK) is the essential upstream protein kinase in the classical $N F-\kappa B$ activating pathway (15-17). In the present study, BMS-345541, a highly selective inhibitor of IKK, was used to explore the role of $\mathrm{NF}-\kappa \mathrm{B}$ in the network of apoptosis $(18,19)$. In the present study, BMS-345541 exhibited a concentration-dependent inhibition of melanoma cell survival in vitro. However, melanoma cells exhibited no greater sensitivity to BMS-345541 than to U0126. When BMS-345541 was used as a co-treatment with U0126, strong synergistic activity was generated, which indicates that combining NF- $\kappa \mathrm{B}$ and MEK inhibition may be a promising approach for treating melanoma with acquired drug resistance.

The molecular mechanism of the U0126-induced antitumor effect and its synergistic effects with BMS-345541 was subsequently explored. The present study demonstrated that U0126 plus BMS-345541 combination treatment enhanced apoptosis, induced cell cycle arrest, and inhibited the expression of Bcl-2. A previous study demonstrated that BMS-345541 results in accumulation of BE-13 and DND-41 cells in the G2/M phase, and that U0126 results in G1/S phase cell cycle arrest in K562 leukemia cells (18). Consistent with these findings, the present study demonstrated that in A375 melanoma cells, BMS-345541 predominantly blocked cells in G2 phase, U0126 mainly blocked cells in G1 phase, and U0126 plus BMS-345541 blocked cells in G1 and G2 phase, and significantly inhibited tumor cell proliferation and consequently induced apoptosis.

The balance of pro-apoptotic (Bax) and anti-apoptotic (Bcl-2) proteins modulates intrinsic cell death following apoptotic insult $(20,21)$. Therefore, Bcl-2 expression is the key step to protect cells from apoptosis in melanoma. It has a crucial role in chemoresistance in various human cancers $(22,23)$. The present study detected expression of Bcl-2 at $24 \mathrm{~h}$ after treatment with the inhibitors. In accordance with previous studies, BMS-345541 and U0126 downregulated expression of Bcl-2, leading to reversal of chemoresistance and enhancement of apoptosis $(24,25)$.

The major question addressed by the current study is whether combination of IKK and MEK inhibitors improves the efficacy of chemotherapy and enhances inhibition of cell proliferation. The present study demonstrates for the first time that U0126 in combination with BMS-345541 inhibits the proliferation of human melanoma cells, and that the combined effect involves G1 and G2 phase arrest, as well as downregulation of Bcl-2 expression. The curative effect of the majority of single agents that target melanoma is insufficient. Thus, based on the results of the current study, we propose that therapy with NF- $\kappa \mathrm{B}$ and ERK pathway inhibitors may become a novel, improved treatment strategy for patients with melanoma.

Previous studies have revealed that activation of the ERK pathway may promote cell cycle progression from G1 to $\mathrm{S}$ phase, eventually modulating the expression of downstream nuclear transcription factors including $\mathrm{NF}-\kappa \mathrm{B}$, activator protein-1 and signal transducer and activator of transcription 3 (26). Furthermore, blocking the phosphorylation of ERK proteins may lead to NF- $\kappa \mathrm{B}$ inactivation (27).

In conclusion, the results of the present study demonstrated that although melanoma cells were no more sensitive to BMS-345541 alone than to U0126 alone, strong synergistic activity was generated by their combination. This may indicate that MEK inhibitor U0126 induces A375 melanoma cell apoptosis through an NF- $\kappa \mathrm{B}$-independent mechanism. However, the exact underlying molecular mechanisms remain to be elucidated.

\section{Acknowledgements}

The present study was supported by the Natural Science Foundation of Shandong Province (grant no. ZR2010HM022).

\section{References}

1. Bai J, Xie X,Lei Y, et al: Ocular albinism type 1 induced melanoma cell migration is mediated through the RAS/RAF/MEK/ERK signaling pathway. Molecular Medicine Reports 10: 491-495, 2014.

2. Aris $M$ and Barrio MM: Combining Immunotherapy with Oncogene- Targeted Therapy:A New Road for Melanoma Treatment. Front Immunol. 6: 46, 2015.

3. Queirolo P, Picasso V and Spagnolo F. Combined BRAF and MEK inhibition for the treatment of BRAF-mutated metastatic melanoma. Cancer Treatment Reviews 4: 1-8, 2015.

4. Tuveson DA, Weber BL and Herlyn M: BRAF as a potential therapeutic target in melanoma and other malignancies. Cancer Cell 4: 95-98, 2003. 
5. Ning L, Xiao J and Min GP: The effects of the MEK inhibito on the proliferation of human melanoma cells. Chin J Dermatovenereology 26: 961-965, 2012 (In Chinese).

6. Acquaviva J, Smith DL, Jimenez JP, et al: Overcoming acquired BRAF inhibitor resistance in melanoma via targeted inhibition of Hsp90 with ganetespib. Mol Cancer Ther 13: 353-363, 2014.

7. Deng LL, Shao YX, Lv HF, Deng HB and Lv FZ: Over-expressing CYLD augments antitumor activity of TRAIL by inhibiting the $\mathrm{NF}-\kappa \mathrm{B}$ survival signaling in lung cancer cells. Neoplasma 59: 18-29, 2012.

8. Song B, Bian Q, Shao CH, Li G, Liu AA, Jing W, Liu R, Zhang YJ, Zhou YQ, Hu XG and Jin G: Ulinastatin reduces the resistance of liver cancer cells to epirubicin by inhibiting autophagy. PLoS One 10: 1-15, 2015.

9. Zhao B, Fang GJ, Zhu J, et al: The computing method of $\mathrm{IC}_{50}$ in determining cell proliferation inhibition rate by MTT method. Anhui Med Pharm J 11: 834-836, 2007 (In Chinese).

10. Yeh YA, Herenyiova M and Weber G: Quercetin: Synergisticaction with carboxamidotriazole in human breast carcinoma cells Life Sci 57: 1285-1292, 1995.

11. Duffy A and Kummar S: Targeting mitogen-activated protein kinase kinase (MEK) in solid tumors. Target Oncol 4: 267-273, 2009.

12. Pratilas CA, Hanrahan AJ, Halilovic E, et al: Genetic predictors of MEK dependence in non-small cell lung cancer. Cancer Res 68 9375-9383, 2008.

13. Sadaria MR, Yu JA, Meng X, et al: Secretory phospholipase A2 mediates human esophageal adenocarcinoma cell growth and proliferation via ERK 1/2 pathway. Anticancer Res 33: 1337-1342, 2013.

14. Walters DM, Lindberg JM, Adair SJ, et al: Inhibition of the growth of patient-derived pancreatic cancer xenografts with the MEK inhibitor trametinib is augmented by combined treatment with the epidermal growth factor receptor/HER2 inhibitor lapatinib. Neoplasia 15: 143-155, 2013.

15. Madonna G, Ullman CD, Gentilcore G, PalmieriG and Ascierto PA $\mathrm{NF}-\kappa \mathrm{B}$ as potential target in the treatment of melanoma. J Trans Med 10: 53, 2012.

16. Wang Y, Zhou Y, Jia G, Han B, Liu J, Teng Y, et al: Shikonin suppresses tumor growth and synergizes with gemcitabine in a pancreatic cancer xenograft model: Involvement of NF- $\mathrm{NB}$ signaling pathway. Biochem Pharmacol 88: 322-333, 2014.

17. Schmid JA and Birbach A: IkappaB kinase beta(IKKbeta/IKK2/IKBKB) - a key molecule in signaling to the transcription factor NF-kappaB. Cytokine Growth Factor Rev 19: 157-165, 2008.
18. Buontempo F, Chiarini F, Bressanin D, et al: Activity of the selective IкB kinase inhibitor BMS-345541 against T-cell acute lymphoblastic leukemia: Involvement of FOXO3a. Cell Cycle 11: 2467-2475, 2012

19. Yang J, Amiri KI, Burke JR, Schmid JA and Richmond A: BMS-345541 targets inhibitor of kappaB kinase and induces apoptosis in melanoma: Involvement of nuclear factor kappaB and mitochondria pathways. Clin Cancer Res 12: 950-960, 2006.

20. Nesic-Taylor O, Cittelly D, Ye Z, et al: Exogenous Bcl-xL fusion protein spares neurons after spinal cord injury. J Neurosci Res 79: 628-637, 2005.

21. Han X, Lu M, Wang S, Lv D and Liu H: Targeting IKK/NF- $\kappa \mathrm{B}$ pathway reduces infiltration of inflammatory cells and apoptosis after spinal cord injury in rats. Neurosci Lett 511: 28-32, 2012.

22. Zhang H, Cai X, Wang Y, et al: microRNA-143, down-regulated in osteosarcoma, promotes apoptosis and suppresses tumorigenicity by targeting Bcl-2. Oncol Rep 24: 1363-1369, 2010.

23. Wu DW, Wu TC, Wu JY, Cheng YW, Chen YC, Lee MC, Chen CY and Lee $\mathrm{H}$ : Phosphorylation of paxillin confers cisplatin resistance in non-small cell lung cancer via activating ERK-mediated Bcl-2 expression. Oncogene 33: 4385-4395, 2014.

24. Berger A, Quast SA, Plötz M, Kammermeier A and Eberle J: Sensitization of melanoma cells for TRAIL-induced apoptosis by BMS-345541 correlates with altered phosphorylation and activation of Bax. Cell Death Dis 4: e477, 2013.

25. Xie J, Jin B, Li DW, Shen B, Cong N, Zhang TZ and Dong P: ABCG2 regulated by MAPK pathways is associated with cancer progression in laryngeal squamous cell carcinoma. Am J Cancer Res 4: 698-709, 2014.

26. Sullivan RJ and Atkins MB: Molecular-targeted therapy in malignant melanoma. Expert Rev Anticancer Ther 9: 567-581, 2009.

27. Zhang H, Zhang S, He H, Zhao W, Ren K, Chen J and Shao RG: RasGAP-derived peptide 38GAP potentiates the cytotoxicity of cisplatin through inhibitions of Akt, ERK and NF- $\kappa \mathrm{B}$ in colon carcinoma HCT116 cells. Cancer Lett 308: 62-70, 2011. 